\title{
Prediction of esophageal varices in patients with HCV-related cirrhosis using albumin- bilirubin, platelets-albumin-bilirubin score, albumin-bilirubin-platelets grade, and GAR
}

\author{
Ayman Alsebaey ${ }^{*}$ (D), Mohamed Amin Elmazaly and Hesham Mohamed Abougabal
}

\begin{abstract}
Background: Development of esophageal varices (EVs) is the main complication of portal hypertension. Early detection prevents variceal bleeding. Baveno VI consensus recommended endoscopy if transient elastography (TE) $>20 \mathrm{kPa}$ and platelets below 150,000/. $\mathrm{mm}^{3}$.

Aim: Assessment of the reliability of the albumin-bilirubin (ALBI), platelets-albumin-bilirubin (PALBI), albumin-bilirubin-platelets (ALBI-PLT) score, and gamma-glutamyl transferase-platelets (GAR) ratio as non-invasive models for prediction of EVs presence and the need for endoscopy in patients with HCV-related cirrhosis.

Methods: HCV-related F4 fibrosis by TE or cirrhosis patients were included $(n=661)$. Full metabolic profile, CBC, ultrasonography, and endoscopy were done.

Results: The average age was 42.89 years mainly males. Patients with EVs had statistically significant $(p<0.05)$ higher TE values, ALBI, ALBI-PLT, and PALBI than those without EVs. Both groups were comparable for GAR. Large varices were statistically $(p<0.05)$ associated with higher ALBI, ALBI-PLT, and PALBI. Both small and large varices had comparable TE and GAR. EVs detection cutoffs (sensitivity, specificity): TE > $20 \mathrm{kPa}(83.64 \%, 91.62 \%), \mathrm{ALBI}>-2.43$ (81.28\%, 74.89\%), ALBI-PLT > 3 (77.34\%, 72.93\%), and PALBI >- 2.28 (62.1\%, 76.4\%). On comparison of the ROCs, TE was better than ALBI $(p<0.05)$, ALBI-PLT, and PALBI. ALBI was better than ALBI-PLT and PALBI. Both ALBI-PLT and PALBI are comparable $(p>0.05)$. Positive indirect hemagglutination of schistosomiasis, portal vein diameter, splenic vein diameter, TE, ALBI, ALBI-PLT, and PALBI were independent predictors of EVs existence. On multivariate analysis, portal vein diameter, TE, and ALBI score were significant.
\end{abstract}

Conclusion: The ALBI, ALBI-PLT, and PALBI are useful predictors of EVs presence and the need of diagnostic endoscopy especially in centers that lack FibroScan.

Keywords: HCV, Esophageal varices, Cirrhosis, Transient elastography, ALBI, PALBI, ALBI-PLT

\section{Background}

Portal hypertension (PHTN) is a pathological increased portal vein pressure. It is defined as hepatic venous pressure gradient (HVPG) $>5 \mathrm{mmHg}$ [1]. Anatomically, the etiology may be prehepatic, e.g., portal vein thrombosis, intrahepatic, e.g., cirrhosis and post-hepatic, e.g., congestive

\footnotetext{
* Correspondence: aymanalsebaey@liver.menofia.edu.eg

Department of Hepatology and Gastroenterology, National Liver Institute, Menoufia University, Shebeen El-Koom, Egypt
}

hepatopathy. The intrahepatic causes can be classified into presinusoidal as schistosomiasis, sinusoidal as viral-related cirrhosis, and postsinusoidal as Budd-Chiari syndrome [2].

Liver cirrhosis is the most important cause of portal hypertension. There are several factors associated with pathogenesis of portal hypertension. There is increased intrahepatic vascular resistance to the portal flow due to sinusoidal capillarization as well as fibrosis-induced distortion of the vasculature. Dynamically, there is contraction 
of the smooth muscles of the blood vessels, hepatic stellate cells around the sinusoids, and the myofibroblasts in the fibrous septae, in response to increased vasoconstrictors, e.g., endothelins, norepinephrine, angiotensin II, cysteinyl leukotrienes and decreased intrahepatic vasodilators as nitrous oxide. Splanchnic vasodilation in response to glucagon, nitrous oxide, prostacyclin, bacterial translocation, and carbon monoxide is a major cause of increased portal venous flow $[1,3]$.

Esophageal varices (EVs), a major complication of portal hypertension, may rupture and bleed with increased mortality rate. EVs are dilated tortuous submucosal veins usually in the distal esophagus. They develop when HVPG > $10 \mathrm{mmHg}$ but bleed when HVPG > $12 \mathrm{mmHg}$ [1]. Endoscopy is the gold standard for the detection and diagnosis for the follow-up of EVs.

By doing endoscopy, we can classify variceal size, detect gastric varices and portal hypertensive gastropathy. Moreover, endoscopy is a therapeutic tool that allows variceal eradication, for example, band ligation and glue obturation of gastric varices [4].

Endoscopy is costly, bothersome for the patients especially if done without conscious sedation. So, noninvasive methods for variceal detection are warranted. Clinically, splenomegaly, platelet count, and platelet to spleen ratio > 909 are suggestive of portal hypertension. Radiologically, Doppler, CT, and MRI can detect varices. Furthermore, liver stiffness measured by transient elastography (TE) as with FibroScan can predict EVs. Recently, endoscopic video capsule can diagnose EVs but cannot assess the size [5].

On the one hand, the Baveno VI consensus [6] recommended screening endoscopy in patients with liver transient elastography (TE) $>20 \mathrm{kPa}$ and platelets $<150,000 /$ $\mathrm{mm}^{3}$ and vice versa. On the other hand, the TE measurement by FibroScan is not available in all hospitals.

In clinical practice, the physician needs models based on the routine investigations to alarm him about the probability of esophageal varices and need of the endoscopy.

This study aimed at assessing albumin-bilirubin (ALBI), platelets-albumin-bilirubin score (PALBI), albumin-bilirubinplatelets grade (ALBI-PLT), and gamma-glutamyl transferaseplatelets (GAR) ratio as non-invasive models for prediction of EVs presence and the need for endoscopy in patients with HCV-related cirrhosis.

\section{Methods}

This study was conducted in the National Liver Institute hospitals, Menoufia University. After patient education and answering for all questions, an informed consent was signed by all patients. The study was approved by the institutional review board.

Six hundred sixty-one HCV patients with F4 fibrosis as measured by transient elastography $(n=423,62.5 \%)$
$[7,8]$ or clinically diagnosed as having cirrhosis. The diagnosis of liver cirrhosis was done depending on the clinical, laboratory and radiological features by abdominal ultrasonography [9].

Exclusion criteria included the following: dual or other liver disease (HBV, alcohol, etc.); portal vein thrombosis; gastric varices on endoscopy; history of previous endoscopy; previous variceal bleeding, ascites; hepatic encephalopathy; and hepatocellular carcinoma.

Thorough history taking and complete clinical examination of the patients were done. Full metabolic profile, $\mathrm{CBC}$, and INR were done. Gamma-glutamyl transferase was done only in 148 cases. All investigations were done within 1 week before endoscopy.

Upper esophageo-gastroscopy was done by the same endoscopist (A) to screen for EVs presence and grade discrimination. EVs were classified into small and large varices [10].

Calculations:

ALBI $=\left(\log _{10}\right.$ bilirubin $\left.\mu \mathrm{mol} / \mathrm{L} \times 0.66\right)+($ albumin $\mathrm{g} / \mathrm{L} \times$ - 0.085) [11].

ALBI grades: ALBI $\mathrm{I} \leq-2.60$, ALBI II $>-2.60$ to $\leq-$ 1.39 and ALBI III $>-1.39$.

PALBI $=\left(2.02 \times \log _{10}\right.$ bilirubin $)+\left(-0.37 \times\left[\log _{10}\right.\right.$ bilirubin $\left.]^{2}\right)+(-0.04 \times$ albumin $)+\left(-3.48 \times \log _{10}\right.$ platelets $)+$ $\left(1.01 \times\left[\log _{10} \text { platelets }\right]^{2}\right)[12,13]$.

PALBI grades: PALBI grade 1: value $\leq-2.53$, PALBI grade 2: value from -2.53 to -2.09 , PALBI grade 3 : value $>-2.09$

ALBI-PLT $=$ sum of the ALBI grade (I-III) to the platelet count grade (I-II). Grade I platelet count $=$ platelets > $150,000 / \mathrm{mm}^{3}$ and grade II platelet count $=$ platelets $\leq 150$, $000 / \mathrm{mm}^{3}$. The ALBI PLT range is $2-5$ [14].

GAR = gamma - glutamyl transferase $(\mathrm{U} / \mathrm{L}) /$ platelets $\mathrm{mm}^{3} \times 100$ [15].

N.B. GAR was calculated for 77 patients without varices, 71 patients with EVs (24 small varices and 47 large varices).

\section{Statistical analysis}

Data were statistically analyzed using $\mathrm{IBM}^{\circ} \mathrm{SPSS}^{\circ}$ Statistics version 21 for Windows (IBM Corporation, North Castle Drive, Armonk, NY, USA) and MedCalc ${ }^{\circ}$ version 18.2.1 (Seoul, Republic of Korea). Data were expressed as mean \pm standard deviation, median (interquartile range) for data that are not normally distributed and column percentage for nominal data. All $p$ values are 2 tailed, with values $<0.05$ considered statistically significant, $p=0.01$ is highly significant and $p=0.001$ is very highly significant. Comparisons between two groups were performed using Student's $t$ test for parametric data, and Mann-Whitney test for non-parametric data. Chi-squared test $\left(\chi^{2}\right)$ and Fisher exact test for categorical data analysis. The receiver operating characteristic 
Table 1 Baseline characteristics, investigations, and scores

\begin{tabular}{|c|c|c|c|c|}
\hline & \multirow{2}{*}{$\begin{array}{l}\text { No EVs } \\
N=458\end{array}$} & \multirow{2}{*}{$\begin{array}{l}\text { EVs } \\
N=203\end{array}$} & \multirow[t]{2}{*}{$p$} \\
\hline & & & & \\
\hline \multicolumn{2}{|l|}{ Age (years) } & $\begin{array}{l}40.48 \pm 11.38 \\
40(17)\end{array}$ & $\begin{array}{l}51.16 \pm 8.55 \\
51(9)\end{array}$ & $0.001^{\#}$ \\
\hline \multirow[t]{2}{*}{ Sex } & Female & $131(28.6 \%)$ & $65(32.0 \%)$ & 0.406 \\
\hline & Male & $327(71.4 \%)$ & $138(68.0 \%)$ & \\
\hline \multirow[t]{2}{*}{ IHA Sch. } & Negative & $320(89.4 \%)$ & $43(78.2 \%)$ & 0.025 \\
\hline & Positive & $38(10.6 \%)$ & $12(21.8 \%)$ & \\
\hline \multicolumn{2}{|c|}{ Total bilirubin (mg/dL) } & $\begin{array}{l}1.26 \pm 1.74 \\
0.85(0.5)\end{array}$ & $\begin{array}{l}2.01 \pm 2.16 \\
1.5(1.4)\end{array}$ & $0.001^{\#}$ \\
\hline \multicolumn{2}{|l|}{ Albumin (mg/dL) } & $\begin{array}{l}3.96 \pm 0.82 \\
4.2(0.8)\end{array}$ & $\begin{array}{l}3.09 \pm 0.81 \\
3.1(1.1)\end{array}$ & $0.001^{\#}$ \\
\hline \multicolumn{2}{|l|}{ AST (U/L) } & $\begin{array}{l}55.43 \pm 41.26 \\
45(34)\end{array}$ & $\begin{array}{l}78.40 \pm 115.99 \\
59(47)\end{array}$ & $0.001^{\#}$ \\
\hline \multicolumn{2}{|l|}{$\mathrm{ALT}(\mathrm{U} / \mathrm{L})$} & $\begin{array}{l}55.59 \pm 48.14 \\
45(34)\end{array}$ & $\begin{array}{l}57.41 \pm 75.28 \\
46(39)\end{array}$ & $0.912^{\#}$ \\
\hline \multicolumn{2}{|l|}{ GGT (U/L) } & $\begin{array}{l}64.4787 .14 \\
32(56.5)\end{array}$ & $\begin{array}{l}63.70 \pm 69.60 \\
34(52)\end{array}$ & $0.338^{\#}$ \\
\hline \multicolumn{2}{|l|}{ Hemoglobin ( $g / d L)$} & $\begin{array}{l}13.48 \pm 1.51 \\
13.5(2.1)\end{array}$ & $\begin{array}{l}12.25 \pm 3.06 \\
12(2.8)\end{array}$ & $0.001^{\#}$ \\
\hline \multicolumn{2}{|l|}{ WBCs $\left(/ \mathrm{mm}^{3}\right)$} & $\begin{array}{l}6.52 \pm 7.16 \\
5.9(3)\end{array}$ & $\begin{array}{l}4.60 \pm 1.44 \\
4.45(2)\end{array}$ & $0.001^{\#}$ \\
\hline \multicolumn{2}{|l|}{ Platelets $\left(10^{9} / \mathrm{L}\right)$} & $\begin{array}{l}175.99 \pm 66.72 \\
175(90)\end{array}$ & $\begin{array}{l}92.42 \pm 39.39 \\
89(54)\end{array}$ & 0.001 \\
\hline \multirow[t]{2}{*}{ Platelets $\left(/ \mathrm{mm}^{3}\right)$} & $\geq 15010^{9} / \mathrm{L}$ & $308(96 \%)$ & $13(4 \%)$ & 0.001 \\
\hline & $<15010^{9} / \mathrm{L}$ & $150(44.1 \%)$ & $190(55.9 \%)$ & \\
\hline \multicolumn{2}{|l|}{ INR } & $\begin{array}{l}1.17 \pm 0.28 \\
1.1(0.2)\end{array}$ & $\begin{array}{l}1.33 \pm 0.28 \\
1.3(0.2)\end{array}$ & $0.001^{\#}$ \\
\hline \multicolumn{2}{|c|}{ Portal vein diameter $(\mathrm{cm})$} & $\begin{array}{l}11.39 \pm 1.53 \\
11(2)\end{array}$ & $\begin{array}{l}13.17 \pm 2.36 \\
13(2)\end{array}$ & $0.001^{\#}$ \\
\hline \multicolumn{2}{|c|}{ Splenic vein diameter $(\mathrm{cm})$} & $\begin{array}{l}9.28 \pm 1.09 \\
9(1)\end{array}$ & $\begin{array}{l}9.60 \pm 1.51 \\
9(1)\end{array}$ & $0.052^{\#}$ \\
\hline \multicolumn{2}{|c|}{ Transient elastography ( $\mathrm{kPa}$ ) } & $\begin{array}{l}17.54 \pm 6.46 \\
16.8(7.9)\end{array}$ & $\begin{array}{l}31.92 \pm 13.29 \\
29.6(16.7)\end{array}$ & $0.001^{\#}$ \\
\hline \multicolumn{2}{|l|}{ ALBI score } & $\begin{array}{l}-2.58 \pm 0.84 \\
-2.84(0.7)\end{array}$ & $\begin{array}{l}-1.71 \pm 0.81 \\
-1.68(1.16)\end{array}$ & $0.001^{\#}$ \\
\hline \multirow[t]{3}{*}{ ALBI grade } & 1 & $310(67.7 \%)$ & $31(15.3 \%)$ & 0.001 \\
\hline & 2 & 91 (19.9\%) & $106(52.2 \%)$ & \\
\hline & 3 & $57(12.4 \%)$ & $66(32.5 \%)$ & \\
\hline \multicolumn{2}{|l|}{ ALBI-PLT score } & $\begin{array}{l}2.83 \pm 1.04 \\
2(2)\end{array}$ & $\begin{array}{l}3.99 \pm 0.88 \\
4(1)\end{array}$ & $0.001^{\#}$ \\
\hline \multirow[t]{4}{*}{ ALBI-PLT grade } & 2 & $249(54.4 \%)$ & $17(8.4 \%)$ & 0.001 \\
\hline & 3 & $85(18.6 \%)$ & $29(14.3 \%)$ & \\
\hline & 4 & $78(17 \%)$ & $96(47.3 \%)$ & \\
\hline & 5 & $46(10 \%)$ & $61(30 \%)$ & \\
\hline \multicolumn{2}{|l|}{ PALBI score } & $-2.46 \pm 0.51$ & $-2.09 \pm 0.55$ & 0.001 \\
\hline \multirow[t]{3}{*}{ PALBI grade } & 1 & $252(55 \%)$ & 46 (22.7\%) & 0.001 \\
\hline & 2 & $128(27.9 \%)$ & 55 (27.1\%) & \\
\hline & 3 & 78 (17\%) & 102 (50.2\%) & \\
\hline \multicolumn{2}{|l|}{ GAR } & $\begin{array}{l}1.82 \pm 5.03 \\
0.83(1.28)\end{array}$ & $\begin{array}{l}1.57 \pm 1.61 \\
0.99(1.2)\end{array}$ & $0.197^{\#}$ \\
\hline
\end{tabular}

$E V s$ esophageal varices, IHA Sch indirect hemagglutination of schistosomiasis "Mann-Whitney test. Data are represented as mean \pm standard deviation, number (percentage) for nominal data and median (interquartile range) for data out of normal distribution
(ROC) curve analysis was used for detection of the cutoff value for the esophageal varices presence and for small versus large varices size discrimination. For each cutoff, sensitivity, specificity, positive predictive value, and negative predictive value were calculated. The ROCs were compared using the DeLong tests to assess variable discrimination. Univariate and multivariate binary logistic regression were done for detecting the predictors of esophageal varices presence irrespective of the size.

\section{Results}

Table 1 demonstrates comparison between patients with and without esophageal varices. Patients with esophageal varices compared to those without varices, were older $(51.16 \pm 8.55$ vs. $40.48 \pm 11.38$ years; $p=0.001)$ and positive for indirect hemagglutination of schistosomiasis (21.8\% vs. $10.6 \% ; p=0.025)$.

Patients with esophageal varices had statistically significant $(p<0.05)$ higher value [median (IQR)] of serum total bilirubin [1.5 (1.4) vs. $0.85(0.5) \mathrm{mg} / \mathrm{dL}]$, serum AST [59 (47) vs. 45 (34) U/L], INR [1.3 (0.2) vs. 1.1 (0.2)], liver stiffness [29.6 (16.7) vs. $16.8(7.9) \mathrm{kPa}$ ], portal vein diameter [13 (2) vs. $11(2) \mathrm{cm}]$, and splenic vein diameter.

Meanwhile, they had statistically significant $(p<0.05)$ lower value [median (IQR)] of serum albumin [3.1 (1.1) vs. $4.2(0.8) \mathrm{mg} / \mathrm{dL}]$, hemoglobin [12 (2.8) vs. $13.5(2.1)$ $\mathrm{g} / \mathrm{dL}], \mathrm{WBCs}\left[4.45(2)\right.$ vs. $\left.5.9(3) \mathrm{mm}^{3}\right]$ and platelets $[89$ (54) vs. $\left.175(90) 10^{9} / \mathrm{L}\right]$. About $55.9 \%$ of the patients with EVs had platelets $<150,000 \mathrm{~mm}^{3}$.

Patients with esophageal varices compared to those without varices (Table 1 and Fig. 1 ) had statistically significant $(p<0.05)$ higher value [median (IQR)] of ALBI score $[-1.68(1.16)$ vs. $-2.84(0.7)]$, ALBI-PLT score [4(1) vs. 2(2)], and PALBI score ( $2.09 \pm 0.55$ vs. -2.46 $\pm 0.51)$. Both groups were comparable as regards GAR $(p=0.197)$.

Table 2 shows discrimination of small and large varices. Large varices were statistically $(p<0.05)$ associated with higher [median (IQR)] liver stiffness [29.8 (14.7) vs. 29 (17.1) kPa], ALBI score [- 1.47 (0.7) vs. - 2.08 (1.1)], ALBI-PLT score [4 (1) vs. 4 (0.5)] and PALBI score [$1.9(0.5)$ vs. $-2.34(0.7)]$. Both small and large varices had comparable GAR $(p=0.535)$.

The ROC curve analysis was used to assess the usefulness of ALBI score, ALBI-PLT score, PALBI score, and GAR as non-invasive models for detection of esophageal varices and discrimination of its grade or size (Table 3).

For variceal detection whatever the size (Figs. 2 and 4); ALBI >- 2.43 had $81.28 \%$ sensitivity, $74.89 \%$ specificity, $58.9 \%$ PPV, and 90\% NPV. The ALBI-PLT score > 3 had 77.34\% sensitivity, $72.93 \%$ specificity, $55.86 \% \mathrm{PPV}$, and $87.90 \%$ NPV. The PALBI score $>-2.28$ had $62.1 \%$ sensitivity, $76.4 \%$ specificity, $53.8 \% \mathrm{PPV}$, and $82 \% \mathrm{NPV}$. In 


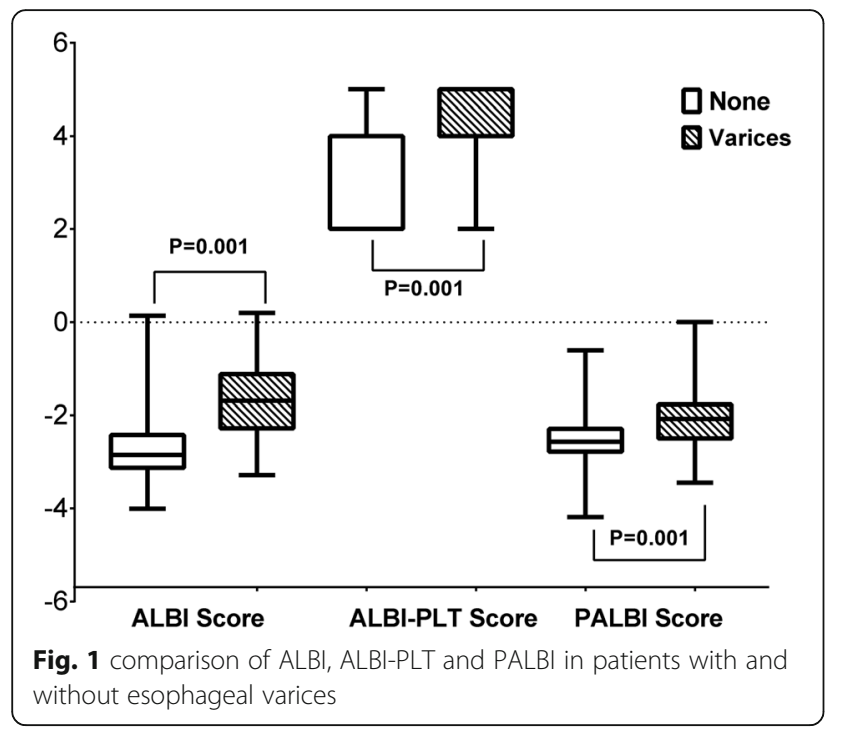

the current study, the cut-off of the liver stiffness adopted by the Baveno VI (>- $20 \mathrm{kPa}$ ) had had $83.64 \%$ sensitivity, $91.62 \%$ specificity, $60.5 \% \mathrm{PPV}$, and $97.3 \%$ NPV.

Pairwise comparison of ROC curves for esophageal varices detection whatever the size revealed TE was better than ALBI $(p<0.05)$, ALBI-PLT, $(p<0.05)$, and PALBI $(p<0.05)$. ALBI was better than ALBI-PLT $(p<$ $0.05)$ and PALBI $(p<0.05)$. Both ALBI-PLT and PALBI are comparable $(p>0.05)$.

For large varices discrimination (Figs. 3 and 4); ALBI >- 1.88 had $92.96 \%$ sensitivity, $60.61 \%$ specificity, $55.9 \%$ PPV, and 94.1\% NPV. The ALBI-PLT score $>4$ had $39.44 \%$ sensitivity, $75 \%$ specificity, $45.9 \%$ PPV, and $69.7 \%$ NPV. The PALBI score $>-2.12$ had $87.32 \%$ sensitivity, 64.39\% specificity, 56.9\% PPV, and 90.4\% NPV.

Table 2 Comparison of TE, ALBI, ALBI-PLT, and PALBI in patients with small and large esophageal varices

\begin{tabular}{|c|c|c|c|}
\hline & \multicolumn{2}{|c|}{ Esophageal varices } & \multirow[t]{3}{*}{$p$} \\
\hline & Small & Large & \\
\hline & $N=132$ & $N=71$ & \\
\hline Transient elastography (kPa) & $\begin{array}{l}30.84 \pm 12.68 \\
29(17.1)\end{array}$ & $\begin{array}{l}33.33 \pm 14.18 \\
29.8(14.7)\end{array}$ & $0.44^{\#}$ \\
\hline ALBI score & $\begin{array}{l}-1.92 \pm 0.83 \\
-2.08(1.1)\end{array}$ & $\begin{array}{l}-1.29 \pm 0.57 \\
-1.47(0.7)\end{array}$ & $0.001^{\#}$ \\
\hline ALBI-PLT score & $\begin{array}{l}3.89 \pm 0.92 \\
4(0.5)\end{array}$ & $\begin{array}{l}4.18 \pm 0.78 \\
4(1)\end{array}$ & $0.039^{\#}$ \\
\hline PALBI score & $\begin{array}{l}-2.24 \pm 0.55 \\
-2.34(0.7)\end{array}$ & $\begin{array}{l}-1.80 \pm 0.43 \\
-1.9(0.5)\end{array}$ & $0.001^{\#}$ \\
\hline GAR & $\begin{array}{l}1.63 \pm 1.64 \\
1.08(1.37)\end{array}$ & $\begin{array}{l}1.54 \pm 1.61 \\
0.86(1.02)\end{array}$ & $0.535^{\sharp}$ \\
\hline
\end{tabular}

"Mann-Whitney test. Data are represented as mean \pm standard deviation and median (interquartile range) for data out of normal distribution
Table 3 Receiver operating characteristic (ROC) curve analysis of TE, ALBI, ALBI-PLT, and PALBI in patients with and without esophageal varices and small versus large varices

\begin{tabular}{|c|c|c|c|c|c|}
\hline \multicolumn{6}{|c|}{ Varices detection } \\
\hline & ALBI & ALBI-PLT & PALBI & $\mathrm{TE}$ & GAR \\
\hline$\overline{A \cup C}$ & 0.794 & 0.784 & 0.708 & 0.956 & 0.562 \\
\hline SE & 0.0184 & 0.0178 & 0.0225 & 0.0106 & \\
\hline$P$ & 0.001 & 0.001 & 0.001 & 0.001 & 0.197 \\
\hline $95 \% \mathrm{Cl}$ & $\begin{array}{l}0.758- \\
0.831\end{array}$ & $\begin{array}{l}0.748- \\
0.821\end{array}$ & $\begin{array}{l}0.664- \\
0.752\end{array}$ & $\begin{array}{l}0.931- \\
0.973\end{array}$ & $\begin{array}{l}0.469- \\
0.654\end{array}$ \\
\hline Cut-off & $>-2.43$ & $>3$ & $>-2.28$ & $>20$ & \\
\hline Sensitivity & 81.28 & 77.34 & 62.1 & 83.64 & \\
\hline Specificity & 74.89 & 72.93 & 76.4 & 91.62 & \\
\hline PPV & 58.9 & 55.86 & 53.8 & 60.5 & \\
\hline NPV & 90 & 87.90 & 82 & 97.3 & \\
\hline \multicolumn{6}{|c|}{ Large varices detection } \\
\hline & ALBI & ALBI-PLT & PALBI & $\mathrm{TE}$ & GAR \\
\hline AUC & 0.744 & 0.582 & 0.754 & 0.561 & 0.545 \\
\hline$P$ & 0.001 & 0.036 & 0.001 & 0.441 & 0.5431 \\
\hline $95 \% \mathrm{Cl}$ & $\begin{array}{l}0.678- \\
0.803\end{array}$ & $\begin{array}{l}0.511- \\
0.651\end{array}$ & $\begin{array}{l}0.688- \\
0.811\end{array}$ & $\begin{array}{l}0.421- \\
0.695\end{array}$ & $\begin{array}{l}0.422- \\
0.663\end{array}$ \\
\hline Cut-off & $>-1.88$ & $>4$ & $>-2.12$ & & \\
\hline Sensitivity & 92.96 & 39.44 & 87.32 & & \\
\hline Specificity & 60.61 & 75 & 64.39 & & \\
\hline PPV & 55.9 & 45.9 & 56.9 & & \\
\hline NPV & 94.1 & 69.7 & 90.4 & & \\
\hline
\end{tabular}

TE transient elastography, $P P V$ positive predictive value, NPV negative predictive value

Pairwise comparison of ROC curves for discrimination of large varices revealed both the ALBI score and PALBI score were comparable $(p=0.526)$. Both ALBI score and PALBI score were better than ALBI-PLT score; $(p=$ $0.001)$ and $(p=0.001)$, respectively.

By univariate logistic regression, the following variables were statistically independent predictors for esophageal varices presence (Table 4); positive indirect hemagglutination of schistosomiasis (odd $=2.35,95 \% \mathrm{CI}$ $=1.14-4.84)$, portal vein diameter $($ odd $=1.71,95 \% \mathrm{CI}=$ $1.52-1.92)$, splenic vein diameter (odd $=1.24,95 \% \mathrm{CI}=$ $1.05-1.45$ ), TE (odds $=1.25,95 \% \mathrm{CI}=1.19-1.32$ ), ALBI score $($ odd $=2.94,95 \% \mathrm{CI}=2.40-3.62)$, ALBI-PLT score (odd $=2.78,95 \% \mathrm{CI}=2.32-3.34)$, and PALBI score (odd $=3.45,95 \% \mathrm{CI}=2.50-4.76)$.

On multivariate analysis, portal vein diameter, TE, and ALBI score were statistically independent predictors for esophageal varices presence.

\section{Discussion}

PHTN and subsequently EVs varices are the main complications of liver cirrhosis. Once the EVs bleed, the hepatic reserve begins to decrease with each attack since the 


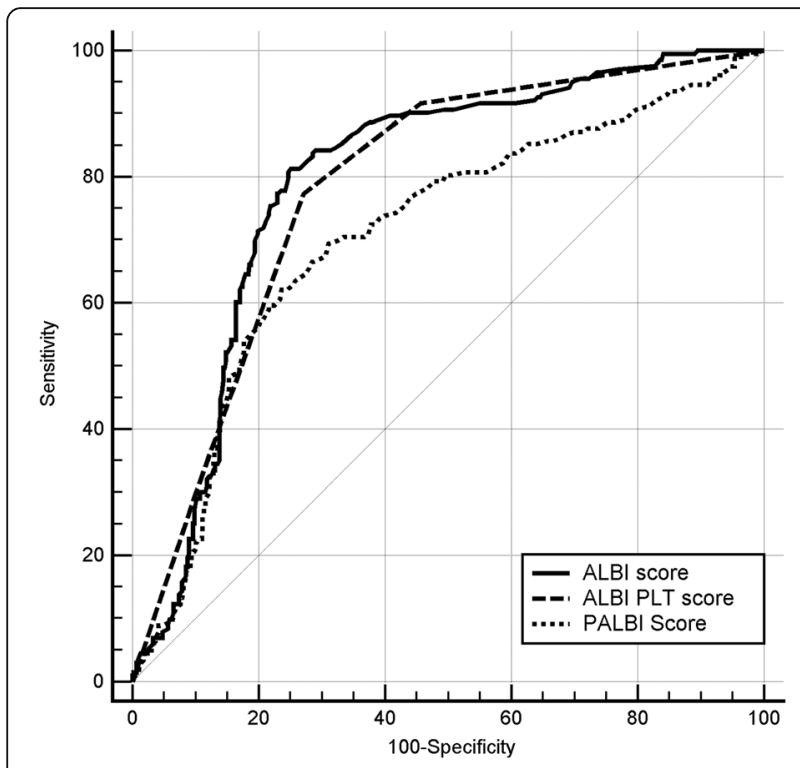

Fig. 2 receiver operating characteristic (ROC) curves analysis of ALBl, ALBI-PLT and PALBI in patients with and without esophageal varices

liver is dependent in such condition on the hepatic artery. Screening for EVs is needed to detect and eradicate them and so prevent variceal bleeding. Endoscopy is the gold standard for the diagnosis but it is invasive maneuver that a lot of patients are afraid to undergo it.

According to the Baveno VI consensus [6], patients with the following criteria can avoid screening endoscopy, namely, $\mathrm{TE}<20 \mathrm{kPa}$ and platelets $>150,000 / \mathrm{mm}^{3}$. These criteria though easily to be applied, depended mainly on the presence of FibroScan that is not available

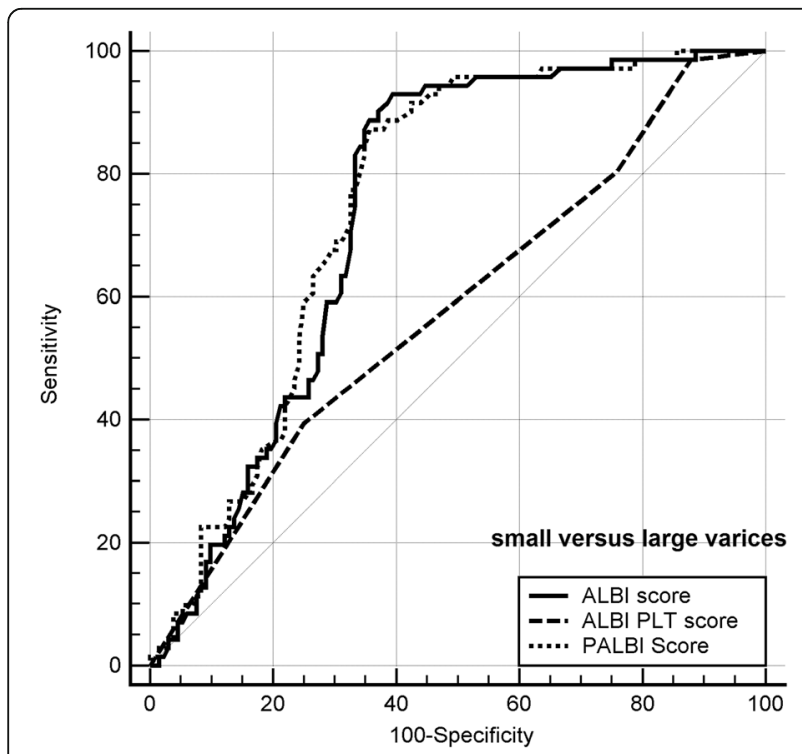

Fig. 3 receiver operating characteristic (ROC) curves analysis of ALBI, ALBI-PLT and PALBI in patients with small versus large varices in all hospitals and primary care units. Another point that the FibroScan is costly regarding the machine price and the maintenance costs. As a result, we still need scores or models that depend on routine laboratory investigations.

Johnson et al. [11] in a large number international study assessed the liver function among patients with hepatocellular carcinoma and correlation with the survival after treatment. They developed the ALBI score that correlated with the liver dysfunction. In comparison with Child Pugh score (CTP), it is simple, non-objective, using two routine labs and being discriminatory for the liver dysfunction. It also correlated with survival. Various publications studied ALBI score in all the aspects of hepatocellular carcinoma from the diagnosis to treatment [16-18].

Roayaie et al. [12, 19] incorporated the platelet count into the ALBI score and called the new model PALBI score. They incorporated the liver function status and the PHTN indirectly. PALBI was divided it into 3 grades (PALBI $1 \leq-2.53$, PALBI $2-2.53$ to -2.09 , PALBI 3 $>-2.09$ ). PALBI score was developed to stratify HCC patient and assessing the survival post-treatment. Liu et al. [20] reinforced the beneficial role of PALBI score.

ALBI and PALBI scores were initiated mainly for hepatocellular carcinoma patients but its success have encouraged researches to assess them in other liver diseases.

Chen et al. [21] found that ALBI score was superior to MELD and CTP score for predicting 1-, 2-, 3-year mortality in HBV-related cirrhosis patients. The lower the ALBI score the more the survival. Shao et al. [22] found that generally ALBI, CTP, and MELD score were comparable for assessing the in-hospital mortality in patients with cirrhosis. In subgroup analysis, CTP score and ALBI score were the best for HBV patients, meanwhile CTP score and MELD were the best for alcoholic hepatitis patients.

Chen and Lin [23] found that high admission ALBI score was a predictor of 3-month mortality in patients with HBV-related acute-on-chronic liver failure. Hou et al. [24] found that patients with hepatic encephalopathy had higher ammonia and ALBI grade, and their combination was useful for predicting advent of encephalopathy. ALBI score was prognostic in patients with primary biliary cholangitis [25]. Both MELD and ALBI predicted the post transjugular intrahepatic portosystemic shunt (TIPS) creation mortality but the performance of MELD score was superior than ALBI score [26].

Xavier et al. [27] conducted a study on 111 patients with acute upper gastrointestinal hemorrhage. Compared to CTP score and MELD score, only ALBI could predict in hospital stay and 30 days mortality. All the 


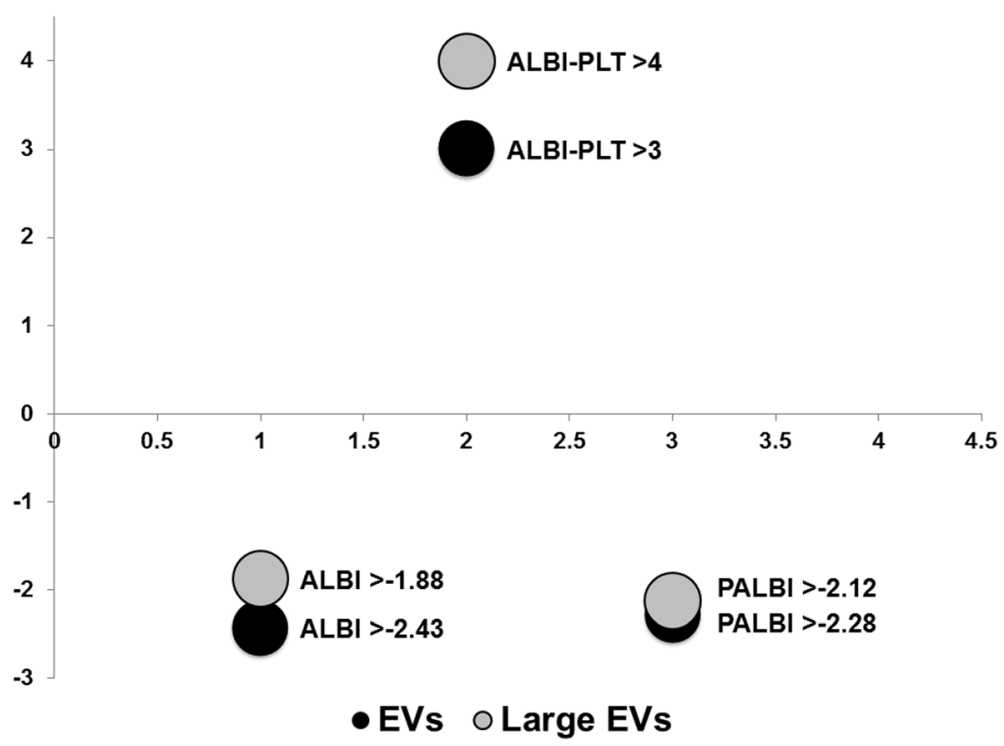

Fig. 4 the cutoffs pf ALBI, ALBI-PLT and PALBI for esophageal varices and large varices discrimination

three score were the same statistically for the 1 year mortality.

Zou et al. [28] found that ALBI score >- 1.492 had $100 \%$ sensitivity, $69.62 \%$ specificity, $7.4 \%$ PPV, and $100 \%$ NPV for predicting acute upper gastrointestinal hemorrhage-related in-hospital mortality of in liver cirrhosis. The etiology of cirrhosis was, HBV, HCV, alcohol, or mixed. Unfortunately, the AUC of ALBI score was statistically comparable to the CTP and MELD score, so it did not add benefit [28].

Recently, Chen et al. [14] developed new score (ALBIPLT) to screen for high-risk esophageal varices (HRVs) in patients with HCC. He combined the ALBI grade (IIII) to platelets grade (I-II) so the sum ranged from 2 to 5. HRVs were common with ALBI grade II > I. ALBIPLT score > 2 had $90 \%$ sensitivity, $27 \%$ specificity, $21 \%$
PPV, and 97\% NPV for detecting HRVs. It is very simple score that also incorporated the dysfunction grade and PHTN indirectly. ABLI-PLT could discriminate patients with HRVs so it is a simple non-invasive screening tool and obviated unnecessary endoscopy.

In the current study, patients with EVs had more incidence of Schistosomiasis $21.8 \%$ against $10.6 \%$. Schistosomiasis per se is a major cause of presinusoidal PHTN but it may augment the effect of HCV on the liver subsequently PHTN $[29,30]$. The increased serum bilirubin, portal vein diameter and the decreased level of serum albumin, hemoglobin, WBCs and platelets reflect liver dysfunction, PHTN, and splenic sequestration or hypersplenism in patients with EVs.

In fact ALBI, PALBI, and ALBI-PT scores could reflect the degree of liver dysfunction and PHTN since higher

Table 4 Univariate and multivariate analysis of predictors of esophageal varices presence

\begin{tabular}{|c|c|c|c|c|c|c|}
\hline & \multicolumn{3}{|c|}{ Univariate } & \multicolumn{3}{|c|}{ Multivariate } \\
\hline & Odds & $95 \% \mathrm{Cl}$ & $p$ & Odds & $95 \% \mathrm{Cl}$ & $p$ \\
\hline Positive IHA Sch. & 2.35 & $1.14-4.84$ & 0.021 & 0.41 & $0.08-1.98$ & 0.264 \\
\hline Portal vein diameter & 1.71 & $1.52-1.92$ & 0.001 & 1.76 & $1.13-2.74$ & 0.012 \\
\hline Splenic vein diameter & 1.24 & $1.05-1.45$ & 0.010 & 0.73 & $0.42-1.27$ & 0.263 \\
\hline Transient elastography & 1.25 & $1.19-1.32$ & 0.001 & 1.21 & $1.14-1.30$ & 0.001 \\
\hline ALBI score & 2.94 & $2.40-3.62$ & 0.001 & 30.19 & $2.37-383.82$ & 0.009 \\
\hline ALBI-PLT score & 2.78 & $2.32-3.34$ & 0.001 & 0.71 & $0.31-1.61$ & 0.407 \\
\hline PALBI score & 3.45 & $2.50-4.76$ & 0.001 & 0.31 & $0.02-5.96$ & 0.438 \\
\hline GAR & 0.98 & $0.90-1.08$ & 0.698 & & & \\
\hline
\end{tabular}

IHA Sch indirect hemagglutination of schistosomiasis 
values were seen in patients with EVs. Furthermore, they were of higher values in patients with large varices compared to small varices.

Despite the promising studies of GAR value in patients with HBV-related fibrosis [15, 20,31], some studies did not find this advantage compared to other score in patients with HBV fibrosis [32, 33]. Shimakawa et al. [34] conducted the first study of GAR in patients with $\mathrm{HCV}$ fibrosis. GAR was comparable to APRI and Fib-4 score. In our study, it was useless for the diagnosis and discrimination of EVs though the number of patient with data was relatively low.

For EVs prediction, ALBI >- 2.43 had $81.28 \%$ sensitivity and $74.89 \%$ specificity. The ALBI-PLT score $>3$ had $77.34 \%$ sensitivity and $72.93 \%$ specificity. The PALBI score $>-2.28$ had $62.1 \%$ sensitivity and $76.4 \%$ specificity.

ALBI >- 1.88 (92.96\% sensitivity, $60.61 \%$ specificity), ALBI-PLT score > 4 (39.44\% sensitivity, $75 \%$ specificity), and PALBI score >- $2.12(87.32 \%$ sensitivity, $64.39 \%$ specificity) could discriminate large from small varices. Again which one is the best? The ALBI-PLT score was less effective than ALBI and PALBI for EVs size discrimination.

The ROC analysis of the TE cutoff ( $>20 \mathrm{kPa}$ ) adopted by the Baveno VI consensus [6] in our study showed 83.64\% sensitivity and $91.62 \%$ specificity. Thrombocytopenia $<150,000 / \mathrm{mm}^{3}$ was statistically associated with EVs but the percentage is not too high (55.9\%).

On comparison of the different ROCs liver stiffness measured by FibroScan was better than all other scores, namely, ALBI, ALBI-PLT, and PALBI. In fact, ALBI was better than ALBI-PLT and PALBI. Both ALBI-PLT and PALBI were comparable.

Regarding the size of the varix, TE did not add benefit unlike the other scores where the ALBI and PALBI were the best for variceal size discrimination.

Positive indirect hemagglutination of schistosomiasis, portal vein diameter, splenic vein diameter, liver stiffness, ALBI score, ALBI-PLT score, and PALBI score were independent predictors of EVs existence. On multivariate analysis, portal vein diameter, TE, and ALBI score were statistically independent predictors for esophageal varices presence.

As aforementioned, three studies were conducted on relationship of ALBI and ALBI-PLT and portal hypertension reflected by variceal bleeding $[27,28]$ or the presence of HRVs [14]. None of them assessed the PALBI score. We are the first study to assess PALBI score and the role of the three scores in screening for EVs in cirrhosis patients without hepatocellular carcinoma.

The cutoff of ALBI-PLT was lower than our study (2 vs. 4-5) [14]. The possible explanations that the authors compared to us chose a cutoff value with high sensitivity
( $90 \%$ vs. $77.34 \%)$ and very low specificity ( $27 \%$ vs. $72.93 \%)$.

The limitations of the study are that it is single-center experience, did not assess the longitudinal follow-up mortality, and did not assess them in non-HCV liver diseases or patients with ascites nor HCC. Large number multi-centric studies are needed.

\section{Conclusion}

The ALBI, ALBI-PLT, and PALBI are useful predictors of EVs presence and the need of diagnostic endoscopy especially in centers that lack FibroScan.

\section{Abbreviations \\ PHTN: Portal hypertension; HVPG: Hepatic venous pressure gradientEVsEsophageal varices; ALBI: Albumin-bilirubin; PALBI: Platelets- albumin-bilirubin; ALBI-PLT: Albumin-bilirubin-platelets; GAR: $\gamma$-Glutamyl transferase-platelets; IHA Sch: Indirect hemagglutination; AUC: Area under the curve; CTP: Child-Pugh; HRVs: High risk esophageal varices; PPV: Positive predictive value; NPV: Negative predictive value; Cl: Confidence interval; TE: Transient elastography}

\section{Acknowledgements}

None

\section{Authors' contributions}

Data collection: AA, HMA, MAE. Study design: AA, HMA, MAE. Manuscript writing and final revision: AA. All authors have read and approved the manuscript

\section{Funding}

None

Availability of data and materials

The datasets used and/or analyzed during the current study are available from the corresponding author on reasonable request.

Ethics approval and consent to participate

It is approved by National Liver Institute IRB 0085/2014. Informed written consent was signed by all patients.

Consent for publication

Not applicable

\section{Competing interests}

None

Received: 13 October 2019 Accepted: 19 March 2020

Published online: 08 May 2020

References

1. Shung DL, Garcia-Tsao G (2017) Liver Capsule: Portal hypertension and varices: pathogenesis, stages, and management. Hepatology 65:1038

2. Treiber G, Csepregi A, Malfertheiner P (2005) The pathophysiology of portal hypertension. Digest Dis 23:6-10

3. Iwakiri Y (2014) Pathophysiology of portal hypertension. Clin Liver Dis 18: 281-291

4. Gulamhusein AF, Kamath PS (2017) The epidemiology and pathogenesis of gastrointestinal varices. Tech Gastrointest Endo 19:62-68

5. de Franchis R, Dell'Era A (2014) Invasive and noninvasive methods to diagnose portal hypertension and esophageal varices. Clin Liver Dis 18:293302

6. de Franchis R (2015) Expanding consensus in portal hypertension: report of the Baveno VI Consensus Workshop: Stratifying risk and individualizing care for portal hypertension. J Hepatol 63:743-752

7. Arena U, Lupsor Platon M, Stasi C, Moscarella S, Assarat A, Bedogni G et al (2013) Liver stiffness is influenced by a standardized meal in patients with 
chronic hepatitis C virus at different stages of fibrotic evolution. Hepatology 58:65-72

8. de Ledinghen V, Vergniol J (2010) Transient elastography for the diagnosis of liver fibrosis. Expert Rev Med Devices 7:811-823

9. Tsochatzis EA, Bosch J, Burroughs AK (2014) Liver cirrhosis. Lancet 383:17491761

10. Lee UE, Friedman SL (2011) Mechanisms of hepatic fibrogenesis. Best Pract Res Clin Gastroenterol 25:195-206

11. Johnson PJ, Berhane S, Kagebayashi C, Satomura S, Teng M, Reeves HL et al (2015) Assessment of liver function in patients with hepatocellular carcinoma: a new evidence-based approach-The ALBI Grade. J Clin Oncol 33:550-558

12. Elshaarawy O, Allam N, Abdelsameea E, Gomaa A, Waked I (2020) Plateletalbumin-bilirubin score - a predictor of outcome of acute variceal bleeding in patients with cirrhosis. World J Hepatol 12(3):99-107

13. Elshaarawy O, Alkhatib A, Elhelbawy M, Gomaa A, Allam N, Alsebaey A et al (2019) Validation of modified albumin-bilirubin-TNM score as a prognostic model to evaluate patients with hepatocellular carcinoma. World J Hepatol 11:542-552

14. Chen P-H, Hsieh W-Y, Su C-W, Hou M-C, Wang Y-P, Hsin IF et al (2018) Combination of albumin-bilirubin grade and platelet to predict compensated patient with hepatocellular carcinoma who do not require endoscopic screening for esophageal varices. Gastrointestinal Endos

15. Lemoine M, Shimakawa Y, Nayagam S, Khali M, Suso P, Lloyd J et al (2016) The gamma-glutamyl transpeptidase to platelet ratio (GPR) predicts significant liver fibrosis and cirrhosis in patients with chronic HBV infection in West Africa. Gut 65:1369-1376

16. Kao W-Y, Su C-W, Chiou Y-Y, Chiu N-C, Liu C-A, Fang K-C et al (2017) Hepatocellular carcinoma: nomograms based on the albumin-bilirubin grade to assess the outcomes of radiofrequency ablation. Radiology 285: 670-680

17. Hiraoka A, Kumada T, Kudo M, Hirooka M, Tsuji K, Itobayashi E et al (2017) Albumin-bilirubin (ALBI) grade as part of the evidence-based clinical practice guideline for HCC of the Japan Society of Hepatology: a comparison with the liver damage and Child-Pugh classifications. Liver Cancer 6:204-215

18. Hiraoka A, Michitaka K, Kumada T, Kudo M (2017) ALBI score as a novel tool in staging and treatment planning for hepatocellular carcinoma: advantage of ALBI grade for universal assessment of hepatic function. Liver Cancer 6: 377-379

19. Hansmann J, Evers MJ, Bui JT, Lokken RP, Lipnik AJ, Gaba RC et al (2017) Albumin-bilirubin and platelet-albumin-bilirubin grades accurately predict overall survival in high-risk patients undergoing conventional transarterial chemoembolization for hepatocellular carcinoma. J Vasc Interv Radiol 28: 1224-31.e2

20. Liu PH, Hsu CY, Hsia CY, Lee YH, Chiou YY, Huang YH et al (2017) ALBI and PALBI grade predict survival for HCC across treatment modalities and BCLC stages in the MELD Era. J Gastroenterol Hepatol 32:879-886

21. Chen RC, Cai YJ, Wu JM, Wang XD, Song M, Wang YQ et al (2017) Usefulness of albumin-bilirubin grade for evaluation of long-term prognosis for hepatitis B-related cirrhosis. J Viral Hepatitis 24:238-245

22. Shao L, Han B, An S, Ma J, Guo X, Romeiro FG et al (2017) Albumin-tobilirubin score for assessing the in-hospital death in cirrhosis. Translat Gastroenterol Hepatol 2:88

23. Chen B, Lin S (2017) Albumin-bilirubin (ALBI) score at admission predicts possible outcomes in patients with acute-on-chronic liver failure. Medicine 96:e7142

24. Hou YL, Gao MD, Guo HY, Wang R, Wang Z, Yu YH et al (2018) Diagnostic value of albumin-bilirubin grade combined with serum ammonia in cirrhosis with hepatic encephalopathy. Zhonghua yi xue za zhi 98:127-131

25. Chan AW, Chan RC, Wong GL, Wong WW, Choi PC, Chan HL et al (2015) New simple prognostic score for primary biliary cirrhosis: Albumin-bilirubin score. J Gastroenterol Hepatol 30:1391-1396

26. Ronald J, Wang Q, Choi SS, Suhocki PV, Hall MD, Smith TP et al (2017) Albumin-bilirubin grade versus MELD score for predicting survival after transjugular intrahepatic portosystemic shunt (TIPS) creation. Diagn Interv Imaging

27. Xavier SA, Vilas-Boas R, Boal Carvalho P, Magalhães JT, Marinho CM, Cotter JB (2018) Assessment of prognostic performance of Albumin-Bilirubin, Child-Pugh, and Model for End-stage Liver Disease scores in patients with liver cirrhosis complicated with acute upper gastrointestinal bleeding. Eur J Gastroenterol Hepatol Publish Ahead of Print
28. Zou D, Qi X, Zhu C, Ning Z, Hou F, Zhao J et al (2016) Albumin-bilirubin score for predicting the in-hospital mortality of acute upper gastrointestinal bleeding in liver cirrhosis: a retrospective study. Turk J Gastroenterol 27:180-186

29. Chofle AA, Jaka H, Koy M, Smart LR, Kabangila R, Ewings FM et al (2014) Oesophageal varices, schistosomiasis, and mortality among patients admitted with haematemesis in Mwanza, Tanzania: a prospective cohort study. BMC Infect Dis 14:303

30. Bahgat MM (2014) Interaction between the neglected tropical disease human schistosomiasis and HCV infection in Egypt: a puzzling relationship. Journal of Clinical and Translational Hepatology 2:134-139

31. Ren T, Wang H, Wu R, Niu J (2017) Gamma-glutamyl transpeptidase-toplatelet ratio predicts significant liver fibrosis of chronic hepatitis B patients in China. 2017:7089702

32. Li Q, Song J, Huang Y, Li X, Zhuo Q, Li W et al (2016) The gamma-glutamyltranspeptidase to platelet ratio does not show advantages than APRI and Fib-4 in diagnosing significant fibrosis and cirrhosis in patients with chronic hepatitis B: a retrospective cohort study in China. Medicine 95:e3372

33. Huang R, Wang G, Tian C, Liu Y, Jia B, Wang J et al (2017) Gamma-glutamyltranspeptidase to platelet ratio is not superior to APRI,FIB-4 and RPR for diagnosing liver fibrosis in CHB patients in China. Sci Rep 7:8543

34. Shimakawa Y, Bonnard P, El Kassas M, Abdel-Hamid M, Esmat G, Fontanet A (2016) Diagnostic accuracy of the $\gamma$-glutamyl transpeptidase to platelet ratio to predict liver fibrosis in Egyptian patients with HCV genotype 4. Gut 65: 1577-1578

\section{Publisher's Note}

Springer Nature remains neutral with regard to jurisdictional claims in published maps and institutional affiliations.

\section{Submit your manuscript to a SpringerOpen ${ }^{\circ}$ journal and benefit from:}

- Convenient online submission

- Rigorous peer review

- Open access: articles freely available online

High visibility within the field

- Retaining the copyright to your article

Submit your next manuscript at $\boldsymbol{\nabla}$ springeropen.com 